Bouba or Kiki with and without vision:

Shape-audio regularities and mental images 


\begin{abstract}
95\% of the world's population associate a rounded visual shape with the spoken word 'bouba', and an angular visual shape with the spoken word 'kiki', known as the bouba/kiki-effect. The bouba/kiki-effect occurs irrespective of familiarity with either the shape or word. This study investigated the bouba/kiki-effect when using haptic touch instead of vision, including the role of visual imagery. It also investigated whether the bouba/kiki shape-audio regularities are noticed at all, that is, whether they affect the bouba/kiki-effect itself and/or the recognition of individual bouba/kiki shapes, and finally what mental images they produce. Three experiments were conducted, with three groups of participants: blind, blindfold, and vision. In Experiment 1, the participants were asked to pick out the tactile/visual shape that they associated with the auditory bouba/kiki. Experiment 1 found that the participants who were blind did not show an instant bouba/kiki-effect (in Trial 1), whereas the blindfolded and the fully sighted did. It also found that the bouba/kiki shape-audio regularities affected the bouba/kiki-effect when using haptic touch: Those who were blind did show the bouba/kiki-effect from Trial 4, and those who were blindfolded no longer did. In Experiment 2, the participants were asked to name one tactile/visual shape and a segment of audio together as either 'Bouba' or 'Kiki'. Experiment 2 found that corresponding shape and audio improved the accuracy of both the blindfolded and the fully sighted, but not of those who were blind - they ignored the audio. Finally, in Experiment 3, the participants were asked to draw the shape that they associated with the auditory bouba/kiki. Experiment 3 found that their mental images, as depicted in their drawings, were not affected by whether they had experienced the bouba/kiki shapes by haptic touch or by vision. Regardless of their prior shape experience, that is, tactile or visual, their mental images included the most characteristic shape feature of bouba and kiki: curve and angle, respectively, and typically not the global shape. When taken together, these experiments suggest that the sensory regularities and mental images concerning bouba and kiki do not have to be based on, or even include visual information.
\end{abstract}

Keywords: bouba/kiki-effect; haptic touch; mental image; multisensory integration; sensory regularities; shape recognition

\title{
Highlights
}

- Shape-audio regularities result in bouba/kiki-effect when blind; not when blindfolded

- Individuals who have no vision integrate information from haptic touch and hearing

- Those who are either blind or fully sighted are able to ignore sensory information

- The blindfolded draw upon visual imagery to solve new problems

- Mental images are not affected by whether hearing is combined with touch or vision

- Shape-audio regularities and mental images do not have to include visual information 


\subsubsection{INTRODUCTION}

95\% of the world's population associate a rounded visual shape with the spoken word 'bouba', and an angular visual shape with the spoken word 'kiki' (cf. Figure 1), known as the bouba/kiki-effect. The bouba/kiki-effect occurs even when people have not had any experience with either the shape or word (Ramachandran \& Hubbard, 2001).

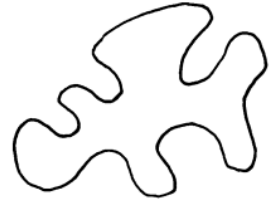

Bouba

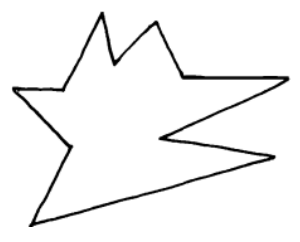

Kiki

Figure 1: Bouba and kiki

These shape-spoken word associations were first reported by Köhler in 1929, who presented two shapes to his participants - one rounded and one angular (similar to those in Figure 1) - and asked them to pick out either 'baluma' or 'takete'. Since then, researchers have found the same associations with other word pairs as well; for example, 'maluma' and 'takete', 'uloomo' and 'takete', 'maa-boo-maa' and 'tuh-kee-tee', and 'bouba' and 'kiki' (e.g. Davis, 1961; Maurer, Pathman, \& Mondloch, 2006; Nielsen \& Rendall, 2011; Ramachandran \& Hubbard, 2001). These shape-spoken word associations were named the 'bouba/kiki-effect' by Ramachandran and Hubbard in 2001. The bouba/kiki-effect precedes language learning and occurs across languages (e.g. Bremner, Caparos, Davidoff, de Fockert, Linnell, \& Spence, 2013; Davis, 1961; Maurer et al., 2006; Ozturk, Krehm, \& Vouloumanos, 2013; Ramachandran \& Hubbard, 2001). It occurs with both bouba and kiki, that is, not just with the curved bouba (cf. people's preference for visual curves over visual angles: e.g., Bar \& Neta, 2006; Bertamini, Palumbo, Gheorghes, \& Galatsidas, 2016; Quinn, Brown, \& Streppa, 1997; Silvia \& Barona, 2009). In fact, the bouba/kiki-effect depends on the particular combination of vowels and consonants [e.g. it does not occur with 'bibi' and 'kuku' (e.g. Nielsen \& Rendall, 2011; Ozturk et al., 2013; Ramachandran \& Hubbard, 2001)]; in fact, it seems that the sound of the word, or its melody, is the most crucial, as opposed to the word itself.

The bouba/kiki-effect appears less robust when using haptic touch instead of vision (Fryer, Freeman, \& Pring, 2014). In haptic touch - the combination of touch and movement (Katz, 1989; Millar, 1997, 2008) - tactile information is perceived serially (Lederman, Browse, \& Klatzky, 1988; Millar, 1984): People who use haptic touch have to link together numerous finger pad-sized pieces of tactile information in order to recognise, for example, each curve in the bouba shape and each angle in the kiki shape.

Fryer, Freeman, and Pring (2014) asked 42 participants who were visually impaired (ranging from congenital to recent onset, and from total blindness to partial sight), and 80 who were fully sighted to explore four bouba and kiki pairs by haptic touch, that is, 2D cut-outs and 3D models based on the original bouba and kiki shapes (Köhler, 1929; Ramachandran \& Hubbard, 2001. Cf. Figure 1): Pairs A and B were 3D and 2D tactile shapes, respectively. Pairs C and D were identical in tactile shape, but differed in tactile texture (smooth $\mathrm{v}$ rough and smooth $\mathrm{v}$ spiky, respectively). All four pairs were presented separately inside a cotton bag, thereby preventing any exploration by vision. Those who were visually impaired picked out the 'correct' tactile bouba/kiki in $\sim 64 \%$ of all trials, and those who were fully sighted in $\sim 90 \%$. Fryer et al. (2014) argued that the significantly less robust bouba/kiki-effect among those who are visually impaired, compared to the fully sighted, is due to a lack of visual imagery. Those who are fully sighted can notice regularities in their 
environment that are not easily accessed with little, or no vision. In a related vein, Fontana (2013) asked 11 blindfolded-sighted participants to grasp a robotic stylus programmed to draw trajectories of the bouba and kiki shapes, and to signal which trajectory they associated with the spoken word 'takete'. After a two-minute kinaesthetic training period with the two bouba and kiki trajectories, $82 \%$ of the participants showed a kinaesthetic-auditory bouba/kiki-effect.

It is still not clear, however, whether there is an instant bouba/kiki-effect when using haptic touch: Fryer et al. (2014) calculated the effect across trials. It is also not clear whether people notice tactile-auditory regularities, and whether these regularities affect the bouba/kiki-effect: Fryer et al. (2014) did not compare the tactile-auditory bouba/kiki-effect on, for example, the first versus the last trial, and Fontana (2013) did not compare the post-training kinaesthetic-auditory bouba/kikieffect to a pre-test, nor to a control group. To this end, it is not clear whether visual imagery in fact is needed for the tactile-auditory bouba/kiki-effect to occur. Those who were fully sighted in Fryer et al.'s (2014) study did not wear a blindfold, and thus could easily observe the experimenter's rounded and angular lip movements when announcing the bouba and kiki words (cf. Ramachandran \& Hubbard, 2001). Inevitably, this brings up the question of whether they in actual fact showed a tactile-visual-auditory bouba/kiki-effect: indeed, perceiving information from three senses simultaneously, instead of drawing upon any visual imagery. Further, in Fryer et al.'s (2014) study, of the 42 participants who were visually impaired only six had congenital total blindness (thus no visual imagery at all), one had congenital visual shape perception, one had congenital face recognition, and 34 had experienced full vision; 13 of whom still had face recognition. In other words; 36 of the 42 participants may have had at least some visual imagery, and 14 of these may have been able to observe at least some of the experimenter's rounded and angular lip movements when announcing the bouba and kiki words (cf. Fryer et al., 2014; Ramachandran \& Hubbard, 2001). This also brings up the question of whether the majority of the participants who were visually impaired in Fryer et al.'s (2014) study failed to draw upon their visual imagery, whether they too in actual fact showed a tactile-visual-auditory bouba/kiki-effect, and/or whether they, for example because of perceiving limited visual information, failed to fully integrate all of the multisensory tactile, visual, and auditory information.

This study, therefore, investigated in three experiments: first, whether there is an instant bouba/kikieffect when using haptic touch, including the role of visual imagery (Experiment 1). It also investigated whether people notice tactile/visual-auditory bouba and kiki regularities, and whether these regularities affect the bouba/kiki-effect (Experiment 1); second, whether these regularities affect the recognition of tactile/visual bouba and kiki shapes (Experiment 2); and third, what mental images these regularities produce, as depicted in tactile/visual drawings (Experiment 3). In order to generate tactile/visual-auditory bouba and kiki regularities (cf. Fryer et al., 2014), and not merely, for example, kiki-shape/kiki-word particularities (Ramachandran \& Hubbard, 2001), this study included two types of tactile/visual (outlined; filled), and two types of auditory bouba and kiki (word; and non-word sound). The three experiments were conducted in fixed order across participants, that is, to keep under control as much as possible the participants' amount and type of experience with the tactile/visual-auditory bouba and kiki regularities (cf. Fryer et al. 2014).

\subsubsection{EXPERIMENT 1: THE BOUBA/KIKI-EFFECT}

This first experiment investigated:

- Is there an instant tactile/visual-auditory bouba/kiki-effect, and how is this bouba/kiki-effect affected by visual imagery?

- Are the tactile/visual-auditory bouba and kiki regularities noticed at all, that is, do they affect the tactile/visual-auditory bouba/kiki-effect?

\subsubsection{Method}

2.1.1.0 Design 
Experiment 1 was designed as a pre-test-post-test quasi-experiment, with three groups of participants: Blind (to which the participants were not randomly assigned), blindfold, and vision. The treatment, or training aimed to generate tactile/visual-auditory bouba and kiki regularities, and thus included a series of repeated and related types of bouba and kiki (outlined; filled and word; sound). In addition, there were three repeated within-group measures, testing the effect of:

- repeated tactile/visual type (outlined) and varied auditory type (word; sound) of bouba and kiki;

- new tactile/visual type (filled) and varied auditory type (word; sound) of bouba and kiki; and,

- tactile/visual-auditory bouba and kiki regularities, that is, varied tactile/visual type (outlined;

filled, and varied auditory type (word; sound) of bouba and kiki.

\subsubsection{Participants}

Thirty-six individuals who were compensated for their time, participated. Twelve were congenitally blind ( 7 females, mean age $=47.1$ years $)$ - ten were born blind and two were blinded less than four months after birth. Of these twelve participants, five had total blindness, three had light perception (perceiving a light source), and four had light projection (perceiving where a light source is situated: WHO, 2017). All were braille readers. Twelve participants were blindfolded-sighted ( 8 females, mean age $=28.3$ years $)$, and twelve were sighted $(11$ females, mean age $=25.1$ years $)$, with normal/corrected to normal vision. All 36 participants had normal/corrected to normal hearing. No one had a cognitive delay or impairment, nor any physical disabilities. Their education ranged from comprehensive school level to a doctoral degree. All were naïve to the bouba/kiki-effect (cf. also Table 1).

Table 1: Self-reported experience in exploring tactile pictures

\begin{tabular}{|l|c|c|c|c|c|}
\hline \multirow{2}{*}{ Participants who were } & \multicolumn{5}{|c|}{ Experience } \\
\cline { 2 - 6 } & $1-$ none & 2 & 3 & 4 & $5-$ a lot \\
\hline congenitally blind & 0 & 1 & 5 & 4 & 2 \\
\hline blindfolded-sighted & 11 & 1 & 0 & 0 & 0 \\
\hline
\end{tabular}

Experience in exploring tactile pictures, symbols, illustrations, graphs, figures, and diagrams (without any vision) produced on swell paper or plastic embossing film, or as a thermoform collage

\subsubsection{Test material}

\subsubsection{Tactile/visual test material}

Bouba and kiki (each shape measuring $30 \times 23 \mathrm{~mm}$ ) were printed on swell paper, glued separately on foam board ( $50 \times 50 \mathrm{~mm} ; 5 \mathrm{~mm}$ thick), and presented on a rubber mat $(140 \times 230 \mathrm{~mm})$. There were two types of bouba and kiki: outlined (1.5 pt. line) and filled (cf. Figure 2). The height of the tactile bouba and kiki shapes were approximately $0.5 \mathrm{~mm}$.

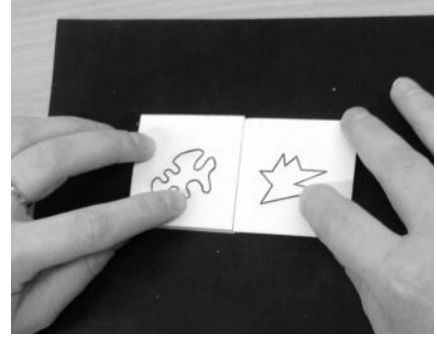

Outlined

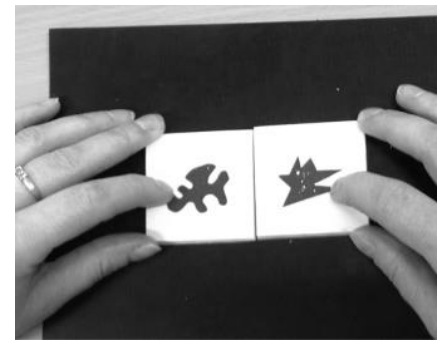

Filled

Figure 2: Tactile/visual test material 


\subsubsection{Auditory test material}

Bouba and kiki were presented separately via an MP3-player, with one built-in loudspeaker. There were two types of bouba and kiki: word and (non-word) sound. The sound was created, using a sine wave generator and pitch bend, by mimicking the melody (i.e. duration and frequency) of the spoken words 'bouba' and 'kiki' (cf. Table 2).

Table 2: Auditory test material

\begin{tabular}{|c|l|l|l|l|}
\hline \multirow{2}{*}{} & \multicolumn{2}{|c|}{ Word: (native) English speaking female } & \multicolumn{2}{c|}{ Sound: Sine wave } \\
\cline { 2 - 5 } & \multicolumn{1}{|c|}{ Duration } & Frequency $($ in $\mathrm{Hz})$ & \multicolumn{1}{c|}{ Duration } & Frequency (in Hz) \\
\hline Bouba & $14.15 / 25.00$ ths of a sec & $759-1500-250$ & $1.00 \mathrm{sec}$ & $474-560$ (centred at 500) \\
\hline Kiki & $18.43 / 25.00$ ths of a sec & $750-250$ & $3.00 \mathrm{sec}$ & $604-1972$ (centred at 1000) \\
\hline
\end{tabular}

\subsubsection{Procedure}

The experiment took place in a quiet room, neutral in colour. All sighted participants were randomly assigned to either the blindfold or the vision-group. The participants who were assigned to the blindfold-group put on their blindfold immediately and prior to the set-up of Experiment 1.

There were eight trials in total: Trials 1-4 contained the outlined type of the tactile/visual bouba and kiki, and Trials 5-8 the filled type. The order of outlined and filled was randomly assigned. Trial 1 (pre-test) was the bouba-word: bouba/kiki was randomly assigned. Both bouba/kiki and word/sound were randomly assigned to the remaining seven trials, so that in total there were four word and four sound trials; four bouba and four kiki trials. The trials were presented in fixed order across participants.

\subsubsection{Set-up of the tactile/visual test material}

The rubber mat was presented directly in front of the participant and prevented the test material from moving around on the table. To enhance the participants' ability to form mental images of the bouba and kiki shapes without interference, for example from their position on the rubber mat, bouba was always on the left, kiki on the right, and they were never rotated. The participants were ignorant of the fixed presentation of bouba and kiki (i.e. bouba on left, kiki on right; no rotation), as well as the two types of bouba and kiki (i.e. outlined and filled).

\subsubsection{Set-up of the auditory test material}

The MP3-player, with one built-in loudspeaker, was presented directly in front of the participant. It prevented the participants in the vision-group from observing the experimenter's lip movements on the bouba/kiki word-trials (cf. Ramachandran \& Hubbard, 2001), thus keeping consistent the amount of sensory information across all experimental groups. The participants were ignorant of the two types of bouba and kiki (i.e. word and sound).

\subsubsection{Conducting the test}

Participants in the blind and blindfold-group were invited to explore the rubber mat (e.g. for size and texture) before the test material was introduced. The experimenter explained that two separate picture cards, each one picturing a tactile/visual shape, would be presented on the rubber mat, and that some audio would correspond to one of the picture cards. All trials were set up behind a carton plate - to ensure that the participants could not take a sneak peek at what was in store. Participants in the blind and the blindfold-group were asked to explore the picture cards by haptic touch, and participants in the vision-group to explore the picture cards by vision. 
At the start of each trial, in the blind and blindfold-group, the participant had to make a fist with both hands and to place them (guided by the experimenter) on top of the presented picture cards, with the experimenter's left hand now being on top. In the vision-group, the carton plate covered the presented picture cards. At this point, the experimenter asked the participant to explore the two tactile/visual shapes: to start when the experimenter's hand/carton plate was removed and to stop by saying 'yes' (upon which the picture cards were covered). Finally, the audio appeared and the participant signalled (by saying e.g. 'left/right'; 'curvy/angular'; 'flower/star'), which tactile/visual shape they associated with the presented audio. The experimenter did not comment on their answer.

\subsubsection{Task}

- Please, pick out the tactile/visual shape that you associate with the presented audio.

\subsubsection{Scoring}

Correct association of the tactile/visual bouba/kiki shape with the presented bouba/kiki audio, for example, the tactile/visual bouba shape associated with the bouba audio.

\subsubsection{Analysis}

Two two-way chi square tests were conducted to compare the three experimental groups (blind; blindfold; vision) before [pre-test (Trial 1)] and after the training [post-test (Trial 8)], that aimed to generate tactile/visual-auditory bouba and kiki regularities. Fisher's exact test was conducted when the number of observations in one or more cells was less than five. In addition, three sets of four chi square goodness-of-fit tests (blind; blindfold; vision) were conducted to test whether there was: - an instant bouba/kiki-effect [pre-test (Trial 1)];

- an effect of repeated tactile/visual type (outlined) and varied auditory type (word; sound) of bouba and kiki [repeated measure (Trial 4)];

- an effect of new tactile/visual type (filled) and varied auditory type (word; sound) of bouba and kiki [repeated measure (Trial 5)]; and,

- an effect of tactile/visual-auditory bouba and kiki regularities, that is, varied tactile/visual type (outlined; filled) and varied auditory type (word; sound) of bouba and kiki [repeated measure and post-test (Trial 8)].

All statistical analyses were calculated on the number of participants, and also when percentages are reported. Because of the modest sample size, the significance level was set to $10 \%$.

\subsubsection{Results and discussion}

$50 \%$ of the participants who were blind, $75 \%$ of those who were blindfolded, and $\sim 83 \%$ of those who were fully sighted showed an instant bouba/kiki-effect before the training that aimed to generate tactile/visual-auditory bouba and kiki regularities [pre-test (Trial 1)]. Fisher's exact test found no significant difference between the participants who were blind and the participants who were blindfolded $(p=0.40)$; between those who were blind and those who were fully sighted ( $p=$ $0.19)$, and between the blindfolded and the fully sighted $(p=1.00)$. The participants who were blind did not show an instant bouba/kiki-effect significantly above the chance level [of $50 \%\left(\chi^{2}(1 . \mathrm{N}=\right.$ $12)=0.00, p=1.00)]$, whereas both the blindfolded and the fully sighted did: $\chi^{2}(1 . \mathrm{N}=12)=3.00$, $p=0.08$ and $\chi^{2}(1 . \mathrm{N}=12)=5.33, p=0.02$, respectively [cf. Figure 3, Trial 1 (pre-test)].

These results are in line with Fryer et al. (2014), who suggested that visual imagery is needed for the tactile-auditory bouba/kiki-effect to occur. Fryer et al. (2014) continued their argument by emphasising the effect of people's ability to notice regularities in their environment. Then again, do the mental images and the sensory regularities in fact have to be based on, or even include visual information? Certainly, Fontana (2013) found a significant kinaesthetic-auditory bouba/kiki-effect after a two-minute kinaesthetic training period with the two bouba and kiki trajectories. 
The question now, therefore, is whether the participants were able to notice tactile/visual-auditory bouba and kiki regularities and, indeed, whether these regularities affected the bouba/kiki-effect.
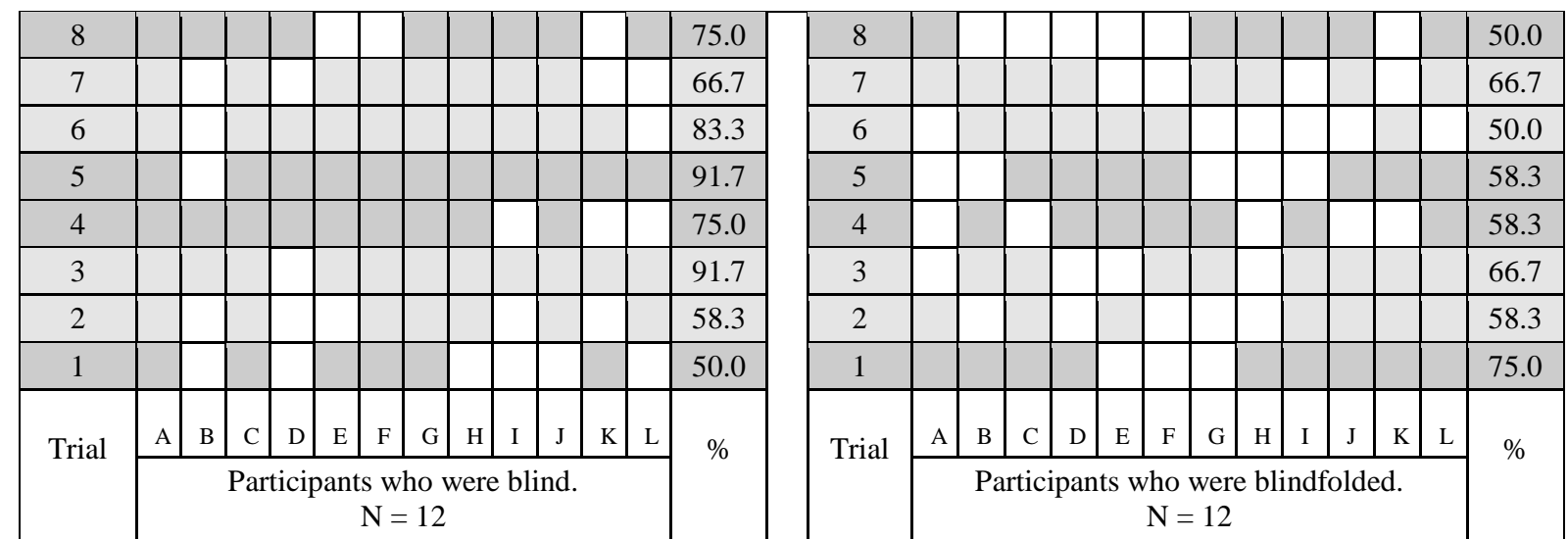

\begin{tabular}{|c|c|c|c|c|c|c|c|c|c|c|}
\hline 8 & & & & & & & & & & 100.0 \\
\hline 7 & & & & & & & & & & 100.0 \\
\hline 6 & & & & & & & & & & 83.3 \\
\hline 5 & & & & & & & & & & 91.7 \\
\hline 4 & & & & & & & & & & 75.0 \\
\hline 3 & & & & & & & & & & 100.0 \\
\hline 2 & & & & & & & & & & 100.0 \\
\hline 1 & & & & & & & & & & 83.3 \\
\hline \multirow{2}{*}{ Trial } & & B & C & $\mathrm{D}$ & $\mathrm{E}$ & \begin{tabular}{l|l|}
$\mathrm{F}$ & $\mathrm{G}$ \\
\end{tabular} & $\mathrm{H}$ & \begin{tabular}{|l|l} 
I & J
\end{tabular} & \begin{tabular}{|l|l}
$\mathrm{K}$ & $\mathrm{L}$ \\
\end{tabular} & \multirow{2}{*}{$\%$} \\
\hline & & & & $\frac{1}{\text { ants }}$ & & $\begin{array}{l}10 \text { were } \\
\mathrm{N}=12\end{array}$ & e fully & ly sight & & \\
\hline
\end{tabular}

Figure 3: The bouba/kiki-effect per participant and trial

Bouba/kiki-effect on pre-test, repeated within-group measures, and post-test

No bouba/kiki-effect

Bouba/kiki-effect on non-tested and non-measured trials

Trials 1-4: Outlined tactile/visual bouba and kiki shapes + auditory:

Trial 1: Bouba word Trial 2: Kiki sound Trial 3: Bouba sound Trial 4: Kiki word

Trials 5-8: Filled tactile/visual bouba and kiki shapes + auditory:

Trial 5: Bouba sound Trial 6: Kiki word Trial 7: Bouba word Trial 8: Kiki sound

Repeated tactile/visual type and varied auditory type of bouba and kiki resulted in a bouba/kiki-effect significantly above the chance level (of 50\%), for the blind and the vision-group. 9 participants in the blind-group, and 9 in the vision-group showed the bouba/kiki-effect: $\chi^{2}(1 . \mathrm{N}=12)=3.00, p=0.08$ and $\chi^{2}(1 . \mathrm{N}=12)=3.00, p=0.08$, respectively. In contrast, 7 participants in the blindfold-group now showed the bouba/kiki-effect $\left[\chi^{2}(1 . \mathrm{N}=12)=0.33, p=0.56\right.$. Cf. Figure 3, Trial 4 (repeated measure) $]$.

Even more participants in the blind and the vision-group showed the bouba/kiki-effect with new tactile/visual type and varied auditory type of bouba and kiki; the blindfold-group remained unchanged. In fact, 11 participants who were blind, and 11 who were fully sighted now showed the 
bouba/kiki-effect: $\chi^{2}(1 . \mathrm{N}=12)=8.33, p=0.00$ and $\chi^{2}(1 . \mathrm{N}=12)=8.33, p=0.00$, respectively, and 7 participants in the blindfold-group $\left[\chi^{2}(1 . \mathrm{N}=12)=0.33, p=0.56\right.$. Cf. Figure 3, Trial 5 (repeated measure)].

Finally, the training that aimed to generate tactile/visual-auditory bouba and kiki regularities, that is, varied tactile/visual type and varied auditory type of bouba and kiki, resulted in a bouba/kiki-effect significantly above the chance level (of 50\%), in the blind and the vision-group, but not in the blindfold-group. $75 \%$ of the participants who were blind, $100 \%$ of those who were fully sighted, and $50 \%$ of those who were blindfolded now showed the bouba/kiki-effect: $\chi^{2}(1 . \mathrm{N}=12)=3.00, p=$ $0.08, \chi^{2}(1 . \mathrm{N}=12)=12.00, p=0.00$, and $\chi^{2}(1 . \mathrm{N}=12)=0.00, p=1.00$, respectively [cf. Figure 3, Trial 8 (repeated measure)]. Fisher's exact test found no significant difference between the participants who were blind and the participants who were fully sighted $(p=0.22)$ and between those who were blind and those who were blindfolded $(p=0.40)$, but it did find a significant difference between the fully sighted and the blindfolded $[p=0.01$. Cf. Figure 3, Trial 8 (post-test)].

In sum, both the participants who were blind and the participants who were fully sighted were able to notice tactile/visual-auditory bouba and kiki regularities. The number of participants who showed the bouba/kiki-effect did indeed increase from the pre-test (Trial 1) to the post-test (Trial 8). These results suggest that the sensory regularities and mental images needed for the tactile-auditory bouba/kikieffect to occur do not have to be based on, or even include visual information. In fact, visual imagery was not enough long term for the blindfold-group. The number of participants who showed the tactile-auditory bouba/kiki-effect decreased significantly from the pre-test (Trial 1) to the posttest (Trial 8) - as if they were able to call upon their visual imagery as a 'one off' (in Trial 1, only).

That visual imagery is not enough conforms with previous research on tactile picture recognition, which has suggested that tactile, rather than visual experience is needed (Graven, 2003; Heller, 1989; Heller, Wilson, Steffen, Yoneyama, \& Brackett, 2003). It is not clear however from previous research, whether this tactile experience should include repeated and specific experience, for example with each bouba and kiki shape, or a variety of experiences with familiar shape features, such as angles and curves within the global shapes, for example within the global bouba and kiki shapes (cf. Graven, 2004, 2005; Ostad, 1989).

Experiment 1 found that repeated tactile/visual and varied auditory [cf. Figure 3, Trial 1 (pre-test) and Trial 4 (repeated measure)], and new/varied tactile/visual and varied auditory experience with bouba and kiki [cf. Figure 3, Trial 4 (repeated measure), Trial 5 (repeated measure), and Trial 8 (repeated measure and post-test)] indeed increased the number of participants who showed the tactile/visual-auditory bouba/kiki-effect. Then again, both the tactile/visual and the auditory variation was quite limited (outlined; filled and word; sound). In addition, the increase in the number of participants who showed the tactile/visual-auditory bouba/kiki-effect was found only with those for whom the training was presented to a sense that they were already experienced in using, that is, haptic touch for the blind-group, and vision for the vision-group. It was not found with those for whom the training was presented to a sense that they were not experienced in using, that is, haptic touch for the blindfold-group (cf. Table 1).

That the participants who were blindfolded were not able to notice tactile/visual-auditory bouba and kiki regularities: the number of participants who showed the bouba/kiki-effect decreasing significantly, from the pre-test (Trial 1) to the post-test (Trial 8), brings up questions about perceptual readiness (Brunner, 1957) and expected information (Spence, Nicholls, \& Driver, 2001). Could it be that these blindfolded participants were perceptually curious in the pre-test (Trial 1) - only one had tried exploring tactile pictures before this study (cf. Table 1) - but that they were not in a state of perceptual readiness for using haptic touch long term? If so, could it also be that as their perceptual 
curiosity dampened, their old habits of expecting visual information started to kick in; and thus, their perceived and expected information did not match?

With Experiment 1 in mind, the question now is whether the tactile/visual-auditory bouba and kiki regularities could be used to solve other problems as well, including whether they affected the recognition of tactile/visual bouba and kiki shapes.

\subsubsection{Experiment 2: Recognising tactile/visual bouba and kiki}

This second experiment investigated:

- Do the tactile/visual-auditory bouba and kiki regularities, including visual imagery, affect the recognition of individual tactile/visual bouba/kiki shapes?

\subsubsection{Method}

\subsubsection{Design}

A within-subject design was employed, with three groups of participants: Bind (to which the participants were not randomly assigned), blindfold, and vision. First, there was one measure of the instant recognition of the tactile/visual-auditory bouba and kiki. Then, there were two repeated within-group measures of whether (a) congruous (e.g. bouba + bouba), and (b) incongruous (e.g. bouba + kiki) tactile/visual + auditory information affected the recognition of the tactile/visual bouba and kiki shapes.

\subsubsection{Participants}

The participants were the same as in Experiment 1.

\subsubsection{Test material}

The test material was the same as in Experiment 1. However, to ensure that the three groups of participants had equal auditory experience with bouba and kiki as much as possible (cf. Fryer et al. 2014; Ramachandran \& Hubbard, 2001), the bouba/kiki word was excluded (cf. Table 2).

\subsubsection{Procedure}

Experiment 2 followed immediately after Experiment 1 . The participants who were blindfolded in Experiment $1 \mathrm{kept}$ their blindfold on, and also during the set-up of Experiment 2.

There were eight trials in total. In half the trials the tactile/visual and the auditory bouba and kiki were congruous, and in the other half they were incongruous. Trial 1 was kiki-shape + kiki-sound: bouba/kiki was randomly assigned. In the remaining seven trials, both bouba/kiki and congruous/incongruous were randomly assigned. The tactile/visual type of bouba and kiki (outlined; filled) was randomly assigned to all eight trials. (Trial 1 was the outlined type.) In total, there were four bouba and four kiki trials; four congruous and four incongruous trials, and four outline and four filled trials. All trials were presented in fixed order across participants. The participants were ignorant of the two types of tactile/visual (i.e. outlined and filled) as well as the one type of auditory (i.e. sound) bouba and kiki, and also the two types of congruousness (i.e. congruous and incongruous).

\subsubsection{Set-up of the tactile/visual and auditory test material}

The set-up of the tactile/visual and auditory test material was the same as in Experiment 1, except that in Experiment 2 only one tactile/visual picture card was presented on the rubber mat - either bouba or kiki.

\subsubsection{Conducting the test}

The test material was presented, and the trials introduced as in Experiment 1. Participants in the blind and the blindfold-group were asked to explore the picture cards by haptic touch, and participants in the vision-group to explore the picture cards by vision. In Experiment 2, the 
experimenter explained that one picture card, picturing a tactile/visual shape, would be presented on the rubber mat together with a segment of audio via the MP3-player: the audio appearing one time per picture card, that is, as soon as the participant started exploring the tactile/visual shape. The experimenter asked the participant to explore the tactile/visual shape and listen to the audio in order to name them 'Bouba' or 'Kiki': one name only (cf. Heller, Calcaterra, Burson, \& Tyler, 1996; Heller \& Gentaz, 2014; Pathak \& Pring, 1989), that is, to start when the experimenter's hand/carton plate was removed, to stop by saying 'yes' (upon which the picture card was covered), and to name them. The experimenter did not comment on the participant's answer.

\subsubsection{Task}

Please, name the tactile/visual shape and the audio either 'Bouba' or 'Kiki': one name only.

\subsubsection{Scoring}

Correct naming of the tactile/visual shape.

\subsubsection{Analysis}

First, three chi square goodness-of-fit tests (blind; blindfold; vision) were conducted to test the instant recognition of the tactile/visual-auditory kiki.

Next, three sets of two chi square goodness-of-fit tests (blind; blindfold; vision) were conducted to test whether (1) congruous, and (2) incongruous tactile/visual + auditory information affected the recognition of the tactile/visual bouba and kiki shapes. In these statistical analyses, the participants were counted as 'correct' when they had recognised $\geq 75 \%$ of all tactile/visual bouba and kiki shapes $\left[\chi^{2}(1 . \mathrm{N}=100)=25.00, p=0.00\right]$.

All statistical analyses were calculated on the number of participants, and also when percentages are reported. Because of the modest sample size, the significance level was set to $10 \%$.

\subsubsection{Results and discussion}

$\sim 92 \%$ of the participants who were blind, $75 \%$ of those who were blindfolded, and $100 \%$ of those who were fully sighted showed an instant recognition of the tactile/visual-auditory kiki - indeed all significantly above the chance level (of 50\%): $\chi^{2}(1 . \mathrm{N}=12)=8.33, p=0.00, \chi^{2}(1 . \mathrm{N}=12)=3.00, p$ $=0.08$, and $\chi^{2}(1 . \mathrm{N}=12)=12.00, p=0.00$, respectively (cf. Figure 4 , Trial 1$)$.

These results suggest that when the participants who were experienced in using either haptic touch or vision have noticed the tactile/visual-auditory bouba and kiki regularities (i.e. the blind and the vision-group: cf. Experiment 1), they were able to use these regularities to solve other problems as well, at least the instant recognition of the tactile/visual-auditory bouba and kiki. Moreover, these results lend support to Experiment 1, suggesting that sensory regularities and mental images concerning bouba and kiki do not have to be based on, or even include visual information. With those participants who were not experienced in using haptic touch (i.e. the blindfold-group), these results suggest that they too were able to notice tactile-auditory bouba and kiki regularities - they simply needed a little more experience - (cf. Graven, 2003, 2004, 2005; Heller, 1989; Heller et al. 2003; Ostad, 1989. Cf. Experiment 1). This again brings up the question about perceptual readiness (Brunner, 1957). Was the blindfold-group now perceptually ready for using haptic touch long term, or was their perceptual curiosity merely rekindled, that is, by the new problem to be solved? Either way, it seems that their perceived and expected information now matched (Spence et al. 2001). Alternatively, these results suggest that the participants who were not experienced in using haptic touch (i.e. the blindfold-group) were able to draw upon their visual imagery every time a new problem needed solving, even if only as a 'one off' solely in Trial 1 (cf. Experiment 1; Figure 4). 
Then again, all participants (blind; blindfold; vision) may have ignored half of the presented sensory information altogether, that is, either the tactile/visual or the auditory kiki. Clearly, it would have been possible for them to recognise kiki using one sense only, for example hearing. The question now, therefore, is whether the tactile/visual-auditory bouba and kiki regularities affected their recognition of the tactile/visual bouba and kiki shapes: more specifically, whether (a) congruous, and (b) incongruous tactile/visual + auditory information affected the recognition of tactile/visual bouba and kiki shapes.

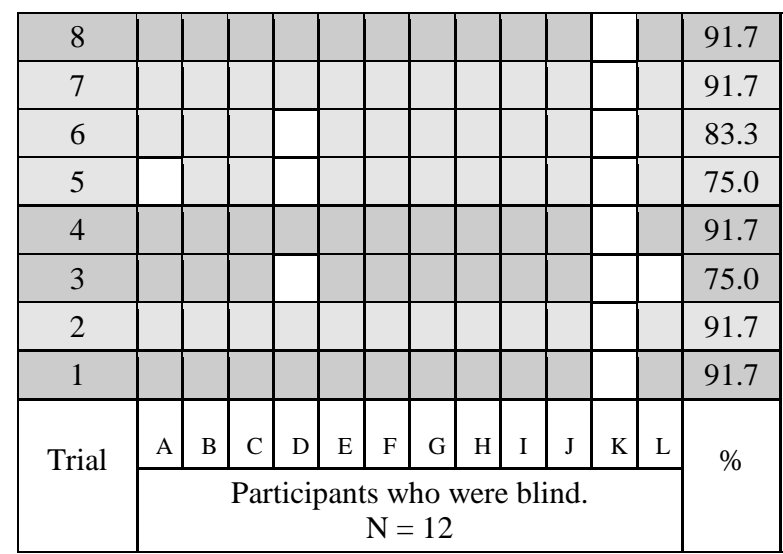

\begin{tabular}{|c|c|c|c|c|c|c|c|c|c|c|c|c|c|}
\hline 8 & & & & & & & & & & & & & 75.0 \\
\hline 7 & & & & & & & & & & & & & 100.0 \\
\hline 6 & & & & & & & & & & & & & 66.7 \\
\hline 5 & & & & & & & & & & & & & 50.0 \\
\hline 4 & & & & & & & & & & & & & 75.0 \\
\hline 3 & & & & & & & & & & & & & 83.3 \\
\hline 2 & & & & & & & & & & & & & 91.7 \\
\hline 1 & & & & & & & & & & & & & 75.0 \\
\hline \multirow{2}{*}{ Trial } & А & В & C & D & E & F & G & H & I & J & K & L & $\%$ \\
\cline { 2 - 4 } & \multicolumn{7}{|c|}{ Participants who were blindfolded. } & \\
\hline
\end{tabular}

\begin{tabular}{|c|c|c|c|c|c|c|c|c|c|c|c|c|}
\hline 8 & & & & & & & & & & & & 100.0 \\
\hline 7 & & & & & & & & & & & & 50.0 \\
\hline 6 & & & & & & & & & & & & 66.7 \\
\hline 5 & & & & & & & & & & & & 75.0 \\
\hline 4 & & & & & & & & & & & & 100.0 \\
\hline 3 & & & & & & & & & & & & 100.0 \\
\hline 2 & & & & & & & & & & & & 66.7 \\
\hline 1 & & & & & & & & & & & & 100.0 \\
\hline \multirow{2}{*}{ Trial } & & $\mathrm{B}$ & $\mathrm{C}$ & $\mathrm{D}$ & $\mathrm{E}$ & $\mathrm{F}$ & $\mathrm{G}$ & $\mathrm{H}$ & I & \begin{tabular}{l|l|}
$\mathrm{J}$ & $\mathrm{K}$ \\
\end{tabular} & $\mathrm{L}$ & \multirow[t]{2}{*}{$\%$} \\
\hline & \multicolumn{11}{|c|}{$\begin{array}{l}\text { Participants who were fully sighted. } \\
\qquad \mathrm{N}=12 .\end{array}$} & \\
\hline
\end{tabular}

Figure 4: The recognition of bouba and kiki per participant and trial

Correct recognition of congruous tactile/visual + auditory bouba and kiki

Incorrect recognition

Correct recognition of incongruous tactile/visual + auditory bouba and kiki

Tactile/visual + auditory:

Trial 1: Kiki + Kiki Trial 2: Kiki + Bouba

Trial 5: Kiki + Bouba

Trial 6: Bouba + Kiki

Trial 3: Bouba + Bouba

Trial 7: Bouba + Kiki

Trial 4: Bouba + Bouba

Trial 8: Kiki + Kiki

11 participants who were blind recognised the tactile bouba and kiki shapes when congruous audio was presented, and 10 participants when incongruous - both the congruous and the incongruous tactile + auditory information significantly above the chance level (of 50\%): $\chi^{2}(1 . \mathrm{N}=12)=8.33, p$ $=0.00$ and $\chi^{2}(1 . \mathrm{N}=12)=5.33, p=0.02$, respectively (cf. Figure 4). Considering that they recognised the tactile bouba and kiki shapes also when incongruous audio was presented, it seems they ignored the auditory information altogether - they rather relied on the tactile information. One reason for this could be that they had no need per se to integrate the tactile and the auditory 
information - they recognised the tactile bouba and kiki shapes regardless - and another that they considered information from haptic touch to be much more reliable than that from hearing. A third reason for ignoring the auditory information could be that they had not yet adopted the tactileauditory bouba and kiki regularities - they had merely noticed them (cf. Experiment 1).

In the blindfold-group, 9 participants recognised the tactile bouba and kiki shapes when congruous audio was presented, and 8 participants when incongruous - the congruous tactile + auditory information significantly above the chance level (of 50\%): $\chi^{2}(1 . \mathrm{N}=12)=3.00, p=0.08$ and $\chi^{2}(1$. $\mathrm{N}=12$ ) $=1.33, p=0.25$, respectively (cf. Figure 4). Clearly, they integrated the sensory information about bouba and kiki. These results suggest that they had now noticed and adopted the tactile-auditory bouba and kiki regularities (cf. Experiment 1), but also that they had little, or no understanding of when to use these regularities and when to ignore sensory information. When the tactile and the auditory information was, or could not be integrated the majority of the blindfold-group sometimes ignored the tactile information, and sometimes the auditory information, as if they were trying out whether to rely on information from haptic touch or on information from hearing (cf. Figure 4). These results again bring up the questions about visual imagery (cf. Fryer et al. 2014) and expected information (Spence et al. 2001). Did the auditory information, when integrated with the tactile information, enable the blindfold-group to draw upon their visual imagery long term, or were their mental images rather based on tactile-auditory information? They did not know about bouba and kiki before this study (cf. Experiment 1). Also, did the auditory information, when integrated with the tactile information, enable them to match their perceived and expected information? Moreover, when the auditory and the tactile information was, or could not be integrated was their trying out of which sense to rely on in actual fact a trying out of which sensory information, if any, would call upon their visual imagery, and/or which sensory information, if any, would match their expected information?

Experiment 2 found that those who were experienced in using haptic touch ignored the auditory information altogether (i.e. the blind-group), and that those who were not experienced in using haptic touch integrated the tactile and the auditory information about bouba and kiki (i.e. the blindfoldgroup). These results are in line with Hötting and Röder (2009), who suggested that the likelihood of multisensory tactile-auditory integration is low in people who are blind, compared to the blindfolded and the fully sighted, because of their enhanced perceptual skills in using both haptic touch and hearing (cf. 'the inverse efficiency principle of multisensory integration': Stein \& Meredith, 1993).

Finally, 12 participants who were fully sighted recognised the visual bouba and kiki shapes when congruous audio was presented, and 6 participants when incongruous - the congruous visual + auditory information significantly above the chance level (of 50\%): $\chi^{2}(1 . \mathrm{N}=12)=12.00, p=0.00$ and $\chi^{2}(1 . \mathrm{N}=12)=0.00, p=1.00$, respectively (cf. Figure 4). Clearly, like the blindfold-group, they too integrated the sensory information about bouba and kiki. These results suggest that they had adopted the visual-auditory bouba and kiki regularities and that they, in contrast to the blindfoldgroup, had an understanding of when to use these regularities and when to ignore sensory information (cf. Experiment 1). When the visual and the auditory information was, or could not be integrated the vision-group split in two, half the vision-group ignored the auditory information, and half the vision-group disregarded the visual information (cf. Figure 4). It seems that with increased experience in using either haptic touch or vision comes a personal preference for which sense to rely on when the tactile/visual and the auditory bouba and kiki information is, or cannot be integrated. Certainly, those who were experienced in using haptic touch relied on haptic touch (i.e. the blind-group); and those who were experienced in using vision relied on either vision or hearing (i.e. the vision-group), whereas those who were not experienced in using haptic touch sometimes relied on haptic touch and sometimes on hearing (i.e. the blindfold-group). 
With Experiment 1 and Experiment 2 in mind, the question now is what mental images the tactile/visual-auditory bouba and kiki regularities have produced, as depicted in tactile/visual drawings.

\subsubsection{Experiment 3: Mental images of auditory bouba and kiki}

This third experiment investigated:

- What mental images do the tactile/visual-auditory bouba and kiki regularities produce, as depicted in tactile/visual drawings, and how are the mental images affected by prior shape experience, that is, tactile or visual, including visual imagery?

\subsubsection{Method}

\subsubsection{Design}

Experiment 3 was designed as a post-test only quasi-experiment, with three groups of participants: Blind (to which the participants were not randomly assigned), blindfold, and vision. The treatment, or training aimed to produce mental images concerning bouba and kiki, and thus included a variety of experiences with the tactile/visual-auditory bouba and kiki regularities: in fact, already presented in Experiment 1 and Experiment 2.

\subsubsection{Participants}

The participants were the same as in Experiment 1 and Experiment 2 (cf. also Table 3).

Table 3: Self-reported experience in drawing tactile pictures

\begin{tabular}{|l|c|c|c|c|c|}
\hline \multirow{2}{*}{ Participants who were } & \multicolumn{5}{|c|}{ Experience } \\
\cline { 2 - 6 } & $1-$ none & 2 & 3 & 4 & $5-$ a lot \\
\hline congenitally blind & 1 & 5 & 3 & 3 & 0 \\
\hline blindfolded-sighted & 12 & 0 & 0 & 0 & 0 \\
\hline
\end{tabular}

Experience in drawing tactile pictures, symbols, illustrations, graphs, figures, and diagrams (without any vision) using plastic embossing film, braille paper or office paper placed on top of a rubber mat

\subsubsection{Test material}

\subsubsection{Tactile/visual test material}

Plastic embossing film for the moon hand frame, white Office Depot paper $\left(80 \mathrm{~g} / \mathrm{m}^{2}\right.$; cut into the A5-format), and one rubber mat $(140 \times 230 \mathrm{~mm})$. In addition, one saddleback style and one black rollerball pen (Uni-ball UM1535 Impact Gel Rollerball 1.0mm tip).

\subsubsection{Auditory test material}

The auditory test material was the same as in Experiment 1 (cf. Table 2).

\subsubsection{Procedure}

Experiment 3 followed immediately after Experiment 2. The participants who were blindfolded in Experiment 1 and Experiment 2 kept their blindfold on, and also during the set-up of Experiment 3. Both bouba/kiki and word/sound were randomly assigned to all trials, so that there were two bouba and two kiki trials; two word and two sound trials - four trials in total, presented in fixed order across participants. (Trial 1 was the kiki-sound.) The participants were ignorant of the two types of auditory bouba and kiki (i.e. word and sound): indeed, also of the fact that Experiment 3 included bouba and kiki.

\subsubsection{Set-up of the tactile/visual and auditory test material}


The set-up of the tactile/visual and auditory test material was the same as in Experiment 1 and Experiment 2, except that in Experiment 3 the plastic embossing film/office paper was presented on top of the rubber mat instead of the tactile/visual picture card(s).

\subsubsection{Conducting the test}

The test material was presented as in Experiment 1 and Experiment 2. In Experiment 3, participants in the blind and the blindfold-group were asked to draw tactile pictures of the presented audio, and participants in the vision-group to draw visual pictures. Participants who were blind and blindfolded were invited to try out and decide for themselves which drawing equipment they preferred - plastic embossing film or office paper and saddleback style or rollerball pen.

The experimenter explained that some audio would be presented via the MP3-player, that is, one time per drawing. All participants had to make a fist with both hands, holding the saddleback style/rollerball pen, and to place them (guided by the experimenter) in the middle of the plastic embossing film/office paper, with the experimenter's left hand now being on top. At this point, the experimenter asked the participant to draw their mental image of the tactile/visual shape that they associated with the presented audio, that is, to start when the experimenter's hand was removed and the audio appeared, and to stop by saying 'yes' (upon which their drawing was removed and labelled with the correct trial number, in the bottom right corner). The experimenter did not comment on the participant's drawing.

\subsubsection{Task}

Please, draw the shape that you associate with the presented audio.

\subsubsection{Scoring}

- What shape is drawn, that is, 'Bouba' or 'Kiki'.

- Who drew the shape, that is, 'somebody who is blind since birth', 'somebody who is blindfolded' or 'somebody who is fully sighted'.

A new group of individuals, naïve to this study, were invited to conduct the scoring. This group consisted of 24 individuals, or scorers (18 females, mean age 52.8 years), all with normal/corrected to normal vision and hearing. All scorers showed a shape-printed word bouba/kiki-effect. The scorers were asked to answer a questionnaire, containing pictures of all the bouba and kiki drawings and two multiple-choice questions per picture: (1) Is the pictured shape 'Bouba' or 'Kiki', and (2) Is the pictured shape drawn by someone who is blind (since birth), blindfolded, or fully sighted.

All drawings were scanned (1:1 size) into the .jpg format and scaled down to include 12 pictures per questionnaire page in landscape A4-format: Bouba/kiki, word/sound, and blind/blindfold/vision were randomly assigned. In total, there were two bouba and two kiki drawings; two word and two sound drawings $\mathrm{x} 12$ pictures drawn by the participants who were blind, 12 by those who were blindfolded, and 12 by those who were fully sighted $=144$ drawings.

Scorers 1, 3, 5, and so on received pictures 1-72, and scorers 2, 4, 6, and so on were given pictures 73-144. Pages 2-8 (containing pictures 1-72) and pages 10-16 (containing pictures 73-144) were shuffled around in random order. The two halves were not mixed. The participants answered the questionnaire in writing, by underlining their chosen answer.

\subsubsection{Analysis}

Two sets of three chi square goodness-of-fit tests (blind; blindfold; vision) were conducted to test whether the participants had drawn (1) instantly, and (2) across trials mental images, that is, tactile/visual shapes, that corresponded to the presented auditory bouba and kiki. Next, a two-way chi square test was conducted to compare the three participant groups (blind; blindfold; vision) 
across trials. Fisher's exact test was conducted when the number of observations in one or more cells was less than five.

In these statistical analyses the participants (blind; blindfold; vision) were counted as 'correspond', 'not correspond', and 'inconclusive', that is:

- 'correspond' when (I) $\geq 9$ scorers agreed on which tactile/visual shape (bouba or kiki) the participant in question had drawn, and (II) this shape corresponded to the presented audio (bouba or kiki) in this trial;

- 'not correspond' when (I) $\geq 9$ scorers agreed on which tactile/visual shape (bouba or kiki) the participant in question had drawn, and (II) this shape did not correspond to the presented audio (bouba or kiki) in this trial; and,

- 'inconclusive' when $<9$ scorers agreed on which tactile/visual shape (bouba or kiki) the participant in question had drawn. 'Inconclusive' participant drawings were removed from the statistical analyses.

- Then, across trials (and with 'inconclusive' participant drawings removed), the participants were counted as 'correspond' when $\geq 75 \%$ of their drawn mental images, that is, tactile/visual shapes, corresponded to the presented auditory bouba and kiki $\left[\chi^{2}(1 . \mathrm{N}=100)=25.00, p=0.00\right]$.

Finally, three chi square goodness-of-fit tests were conducted to test whether the three groups of participants (blind; blindfold; vision) were recognisable across trials.

In these statistical analyses the participants (blind; blindfold; vision) were counted as 'recognised', 'not recognised', and 'inconclusive', that is:

- 'recognised' when (I) $\geq 8$ scorers agreed on who of the participants had drawn the pictured shape in question (blind, blindfold or vision), and (II) this was correct in this trial;

- 'not recognised' when (I) $\geq 8$ scorers agreed on who of the participants had drawn the pictured shape in question (blind, blindfold or vision), and (II) this was not correct in this trial; and,

- 'inconclusive' when $<8$ scorers agreed on who of the participants had drawn the picture shape in question (blind, blindfold or vision). 'Inconclusive' participant drawings were removed from the statistical analyses.

- Then, across trials (and with 'inconclusive' participant recognitions removed), the participants were counted as 'recognised' when recognised in $\geq 75 \%$ of all trials $\left[\chi^{2}(1 . \mathrm{N}=100)=25.00, p=0.00\right]$.

All statistical analyses were calculated on the number of participants, and also when percentages are reported. Because of the modest sample size [both participants (blind; blindfold; vision) and scorers], the significance level was set to $10 \%$.

\subsubsection{Results and discussion}

$\sim 73 \%$ of the participants who were blind, $\sim 90 \%$ of those who were blindfolded, and $80 \%$ of those who were fully sighted instantly drew a mental image, that is, a tactile/visual shape, that corresponded to the auditory kiki - those who were either blindfolded or fully sighted significantly above the chance level (of 50\%): $\chi^{2}(1 . \mathrm{N}=11)=2.27, p=0.13, \chi^{2}(1 . \mathrm{N}=9)=5.44, p=0.02$, and $\chi^{2}(1 . \mathrm{N}=10)=3.60, p=0.06$, respectively (cf. Figure 5, Trial 1).

These results suggest that visual imagery affected what mental images the tactile/visual-auditory bouba and kiki regularities had produced: those who could call upon visual imagery instantly drew a tactile/visual shape that clearly corresponded to the presented auditory kiki (i.e. the blindfold and the vision-group), whereas those who could not call upon visual imagery did not (i.e. the blindgroup). Again, the participants who were blindfolded were able to draw upon their visual imagery when a new problem needed solving (cf. Experiment 1; Experiment 2). Then again, considering that 
the participants who were blind had limited experience in drawing pictures (cf. Table 3), it could be argued that the lack of drawing experience rather than the lack of visual imagery affected their drawing; thus, whether their mental image of the auditory kiki depicted in the drawing could be recognised as the tactile/visual kiki shape.

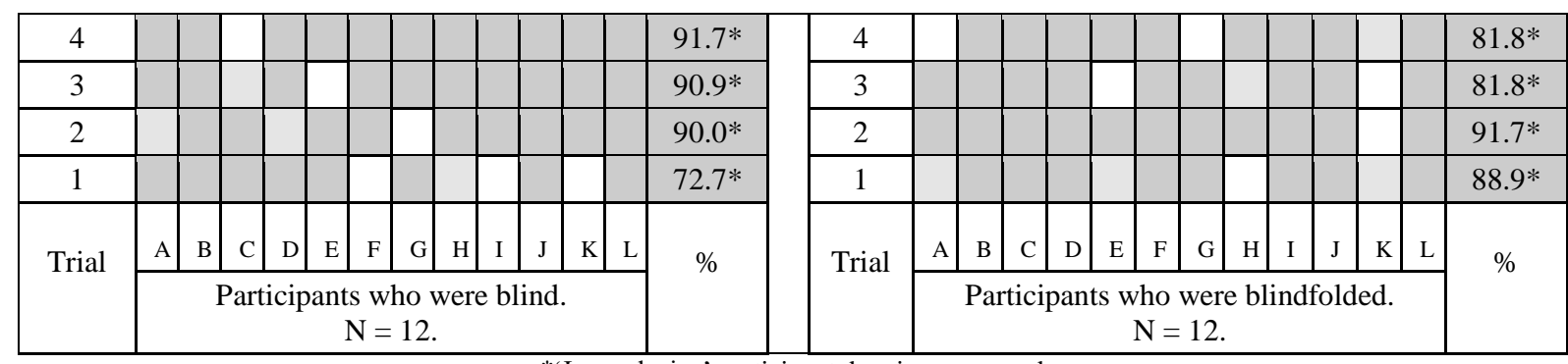

*'Inconclusive' participant drawings removed

\begin{tabular}{|c|c|c|c|c|c|c|c|c|c|c|c|c|c|}
\hline 4 & & & & & & & & & & & & & $90.9 *$ \\
\hline 3 & & & & & & & & & & & & & $100.0^{*}$ \\
\hline 2 & & & & & & & & & & & & & $100.0^{*}$ \\
\hline 1 & & & & & & & & & & & & & $80.0^{*}$ \\
\hline \multirow{2}{*}{ Trial } & $\mathrm{A}$ & B & $\mathrm{C}$ & $\mathrm{D}$ & $\mathrm{E}$ & $\mathrm{F}$ & $\mathrm{G}$ & $\mathrm{H}$ & $\mathrm{I}$ & $\mathrm{J}$ & & $\mathrm{L}$ & \multirow{2}{*}{$\%$} \\
\hline & \multicolumn{12}{|c|}{$\begin{array}{l}\text { Participants who were fully sighted. } \\
\qquad \mathrm{N}=12 .\end{array}$} & \\
\hline
\end{tabular}

Figure 5: The mental image of auditory bouba and kiki per participant and trial

'Correspond' - drawn mental image, that is, tactile/visual shape, $=$ presented audio

'Not correspond' - drawn mental image, that is, tactile/visual shape, $\neq$ presented audio

'Inconclusive' - drawn mental image, that is, tactile/visual shape, ? presented audio

Auditory bouba and kiki:

Trial 1: Kiki sound Trial 2: Bouba word Trial 3: Bouba sound Trial 4: Kiki word

Across trials, 11 participants who were blind drew mental images, that is, tactile/visual shapes, that corresponded to the presented audio, 8 participants who were blindfolded, and 12 who were fully sighted - those who were either blind or fully sighted significantly above the chance level (of 50\%): $\chi^{2}(1 . \mathrm{N}=12)=8.33, p=0.00,{ }^{2}(1 . \mathrm{N}=12)=1.33, p=0.25$, and $\chi^{2}(1 . \mathrm{N}=12)=12.00, p=0.00$, respectively (cf. Figure 5). Fisher's exact test found no significant difference between the participants who were blind and the participants who were blindfolded $(p=0.32)$ and between those who were blind and those who were fully sighted $(p=1.00)$, but it did find a significant difference between the blindfolded and the fully sighted ( $p=0.09$. Cf. Figure 5).

Indeed, these results support the above argument that the participants who were blind needed slightly more drawing experience, rather than visual imagery. In fact, they suggest that also these participants had adopted the tactile-auditory bouba and kiki regularities. This brings up the question of whether they in actual fact had adopted these regularities earlier on (cf. Experiment 1; Experiment 2) and that they, like the participants who were fully sighted, had an understanding of when to use these regularities and when to ignore sensory information (cf. Experiment 2). Moreover, these results again suggest that visual imagery was not enough long term for the participants who were blindfolded - they were able to call upon their visual imagery in Trial 1, but not across trials (cf. 
Experiment 1; Experiment 2; Figure 5). This brings up the question of whether their mental images of bouba and kiki were based on tactile, visual, and/or auditory information (cf. Experiment 2).

In sum, and in line with Experiment 1 and Experiment 2, these results suggest that the sensory regularities and mental images concerning bouba and kiki do not have to be based on, or even include visual information (cf. also Figure 6).

\section{Kiki sound}

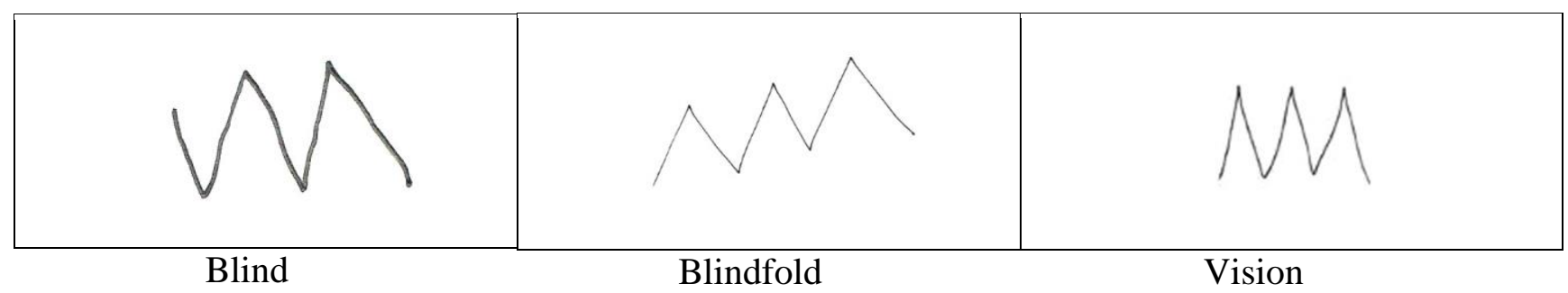

\section{Bouba word}

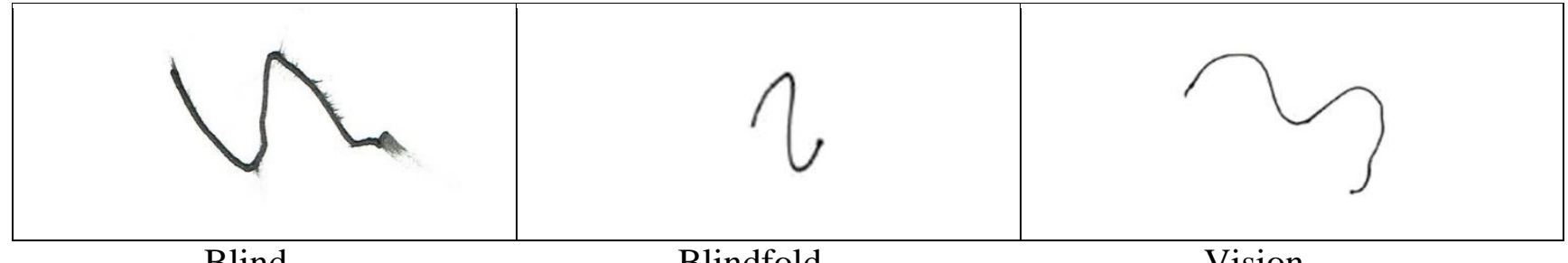

Blind

Blindfold

Vision

\section{Bouba sound}

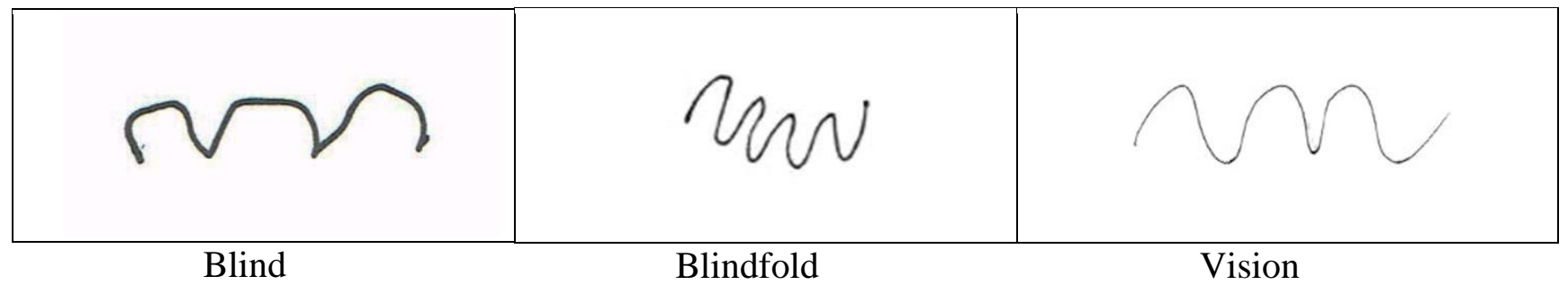

Kiki word

\begin{tabular}{|c|c|c|}
\hline & & \\
\hline
\end{tabular}

Figure 6: Drawings of auditory bouba and kiki: Examples

Interestingly, Experiment 3 found that $\sim 83 \%$ of all mental images, as depicted in the tactile/visual drawings (blind; blindfold; vision), included the most characteristic shape feature of the global bouba and kiki shapes: curve and angle, respectively. They differed in the quantity of repeated 
shape features (e.g. five and eight curves for the bouba-sound: cf. Figure 6) and in direction (horizontal; vertical; diagonal), but typically did not include the global bouba or kiki shape (cf. Figure 1; Figure 6). $17 \%$ of all mental images, as depicted in the tactile/visual drawings, included the global bouba and kiki shapes. It is worth noticing that four participants (two blindfolded; two fully sighted) drew $60 \%$ of these mental images, that is, tactile/visual global shapes. ( $\geq 75 \%$ of their mental images included the global bouba and kiki shapes.) Moreover, one participant who were blind drew KI for the kiki word (cf. Fryer et al. 2014).

Hence, it could be argued that the tactile/visual-auditory bouba and kiki regularities had truly produced mental images. The majority of the participants (blind; blindfold; vision) had not merely copied a memory of the tactile/visual bouba and kiki shapes (cf. Figure 1; Figure 6). In addition, it seems that whichever sense was used in Experiment 1 and Experiment 2 to explore the bouba and kiki shapes - haptic touch or vision - did not affect what mental images the tactile/visual-auditory bouba and kiki regularities had produced.

The question now, therefore, is whether it could be recognised who had drawn the mental image, that is, the tactile/visual shape, of the presented auditory bouba and kiki - 'somebody who is blind since birth, somebody who is blindfolded, or somebody who is fully sighted'.

The participant who had drawn the pictured bouba and kiki shapes (blind; blindfold; vision) was recognised in $\sim 43 \%$ of all the scores' answers. Across trials, 5 participants who were blind (since birth) were recognised, 3 who were blindfolded, and 5 who were fully sighted - indeed no participant group significantly above the chance level (of 33.3\%): $\chi^{2}(1 . \mathrm{N}=12)=0.38, p=0.54$, $\chi^{2}(1 . \mathrm{N}=12)=0.38, p=0.54$, and $\chi^{2}(1 . \mathrm{N}=12)=0.38, p=0.54$, respectively (cf. Figure 7$)$.

\begin{tabular}{|c|c|c|c|c|c|c|c|c|c|c|c|c|c|c|c|c|c|c|c|c|c|c|}
\hline 4 & & & & & & & & & & & 4 & & & & & & & & & & & \\
\hline 3 & & & & & & & & & & & 3 & & & & & & & & & & & \\
\hline 2 & & & & & & & & & & & 2 & & & & & & & & & & & \\
\hline 1 & & & & & & & & & & & 1 & & & & & & & & & & & \\
\hline \multirow{2}{*}{ Trial } & $\mathrm{A}$ & B & $\mathrm{C}$ & $\mathrm{D}$ & $\mathrm{E}$ & $\mathrm{G}$ & $\mathrm{H}$ & I & $\mathrm{K}$ & $\mathrm{L}$ & \multirow{2}{*}{ Trial } & A & B & $\mathrm{C}$ & D & E & $\mathrm{F}$ & $\mathrm{G}$ & $\mathrm{H}$ & $\mathrm{J}$ & $\mathrm{K}$ & L \\
\hline & \multicolumn{10}{|c|}{$\begin{array}{l}\text { Participants who were blind. } \\
\qquad N=12 .\end{array}$} & & \multicolumn{11}{|c|}{$\begin{array}{l}\text { Participants who were blindfolded. } \\
\qquad \mathrm{N}=12 .\end{array}$} \\
\hline
\end{tabular}

\begin{tabular}{|c|c|c|c|c|c|c|c|c|c|c|c|}
\hline 4 & & & & & & & & & & & \\
\hline 3 & & & & & & & & & & & \\
\hline 2 & & & & & & & & & & & \\
\hline 1 & & & & & & & & & & & \\
\hline Trial & $\mathrm{A}$ & В & $\mathrm{C}$ & $\mathrm{D}$ & $\mathrm{E}$ & $\mathrm{F}$ & $\mathrm{G}$ & $\mathrm{H}$ & \begin{tabular}{l|l} 
I & J
\end{tabular} & $\mathrm{K}$ & 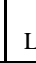 \\
\hline & & & & ats & & $\begin{array}{l}10 \mathrm{~W} \\
\mathrm{~N}=\end{array}$ & $\begin{array}{l}\text { vere } \\
12 .\end{array}$ & full & ly sig & hted. & \\
\hline
\end{tabular}

Figure 7: The recognition of who had drawn bouba and kiki per participant and trial

'Recognised'

'Not recognised'

'Inconclusive' - not clear who had drawn the mental image, that is, tactile/visual bouba/kiki shape

Auditory bouba and kiki:

Trial 1: Kiki sound Trial 2: Bouba word Trial 3: Bouba sound Trial 4: Kiki word 
These results confirm the above suggestion, that whichever sense the participants used in Experiment 1 and Experiment 2 to explore the bouba and kiki shapes - haptic touch or vision - did not affect what mental images the tactile/visual-auditory bouba and kiki regularities had produced. The three groups of participants (blind, blindfold, and vision) were not recognisable across trials.

\subsubsection{General discussion}

This study investigated in three experiments: first, whether there is an instant bouba/kiki-effect when using haptic touch, including the role of visual imagery (Experiment 1). It also investigated whether people notice tactile/visual-auditory bouba and kiki regularities, and whether these regularities affect the bouba/kiki-effect (Experiment 1); second, whether these regularities affect the recognition of tactile/visual bouba and kiki shapes (Experiment 2); and third, what mental images these regularities produce, as depicted in tactile/visual drawings (Experiment 3). In order to generate tactile/visual-auditory bouba and kiki regularities (cf. Fryer et al., 2014), and not merely, for example, kiki-shape/kiki-word particularities (Ramachandran \& Hubbard, 2001), this study included two types of tactile/visual (outlined; filled), and two types of auditory bouba and kiki (word; and non-word sound). The three experiments were conducted in fixed order across participants, that is, to keep under control as much as possible the participants' amount and type of experience with the tactile/visual-auditory bouba and kiki regularities (cf. Fryer et al. 2014). Three groups of participants were included: blind (with no visual imagery at all), blindfold, and vision.

In Experiment 1, the participants were asked to pick out the tactile/visual shape that they associated with the auditory bouba/kiki; in Experiment 2, to name one tactile/visual shape and a segment of audio together as either 'Bouba' or 'Kiki'; and in Experiment 3, to draw the shape that they associated with the auditory bouba/kiki. The participants who were blind did not show an instant tactile/visual-auditory bouba/kiki-effect in Experiment 1, whereas the blindfolded and the fully sighted did. All participants noticed the tactile/visual-auditory bouba and kiki regularities: those who were blind adopted these regularities for Experiment 1, showing the tactile-auditory bouba/kiki-effect from Trial 4; but not for Experiment 2, recognising the tactile bouba and kiki shapes - they rather ignored the auditory information. Those who were blindfolded were able to draw upon their visual imagery for every new problem to be solved, but not long term, that is, in all three experiments. They adopted the tactile-auditory bouba and kiki regularities for Experiment 2, clearly integrating tactile and auditory information, but not for Experiment 1 - the number of participants who showed the tactile-auditory bouba/kiki-effect decreased from the pre-test (Trial 1) to the post-test (Trial 8). Those who were fully sighted had noticed the visual-auditory bouba and kiki regularities before this study, clearly showing the visual-auditory bouba/kiki-effect in Experiment 1. They adopted these regularities for Experiment 2, but also had an understanding of when to ignore sensory information - half of them ignored the auditory information, and half of them the visual information. Finally, the participants' mental images of bouba and kiki, as depicted in their drawings (Experiment 3), were not affected by whether they had experienced the bouba/kiki shapes by haptic touch or by vision (in Experiment 1 and Experiment 2). Regardless of their prior shape experience, that is, tactile or visual, their mental images included the most characteristic shape feature of bouba and kiki: curve and angle, respectively, and typically not the global shape.

Interestingly, all participants noticed and adopted the tactile/visual bouba and kiki regularities otherwise they would not have been able to solve the task in Experiment 1 and Experiment 3 -; however, only those who were experienced in using either haptic touch or vision also knew when to ignore sensory information [i.e. the blind and the vision-group (cf. Experiment 2)]. These results stand in contrast to Hötting and Röder (2009), who suggested that the likelihood of multisensory tactile-auditory integration is low in people who are blind, that is, because of their enhanced perceptual skills in using both haptic touch and hearing (cf. 'the inverse efficiency principle of 
multisensory integration': Stein \& Meredith, 1993). According to Hötting and Röder's (2009) suggestion, the participants who were blind in this study failed to integrate the multisensory tactileauditory information about bouba and kiki (cf. Experiment 2) - they did not know when to ignore sensory information. Then again, Hötting and Röder's (2009) suggestion is based on previous research on passive touch and hearing: They investigated (2004) whether people are able to perceive the correct number of tactile stimuli (from one to four) presented onto their right index finger regardless of the number of auditory tones (from none to four). Further research is needed to investigate whether auditory information affects haptic touch and passive touch differently.

In either case, that is, either ignoring or failing to integrate sensory information; could it be that the perceiving and processing of tactile information and the perceiving and processing of auditory information did not match, for example, in speed? Did, for example, haptic touch need more time to perceive and process the tactile bouba and kiki shapes than hearing did to perceive and process the audio? Indeed, haptic touch had to actively perceive and link together numerous finger pad-sized pieces of tactile information (cf. Katz, 1989; Lederman et. al., 1988; Millar, 1984, 1997, 2008), whereas hearing perceived auditory information delivered as a complete bouba/kiki word or sound (cf. Table 2). More in this vein, could it be that haptic touch failed to perceive all the pieces of the tactile bouba/kiki shape and/or to link the pieces together correctly? This may, in fact, explain why the participants who were blind in this study did not show an instant bouba/kiki-effect [in Experiment 1, pre-test (Trial 1)], and also why they did not instantly draw a recognisable picture of their mental image of the presented audio [in Experiment 3, Trial 1. People who are congenitally blind typically use one hand to draw, e.g. the shape, and the other to follow their drawn line, i.e. behind the stylus/pen, and/or both hands to 'double-check' their finished drawing)]. Further research is needed to investigate whether information from haptic touch and hearing would be integrated, instantly, if haptic touch activated the audio as it explored the tactile information - piece-by-piece-by-piece. Moreover, could it be that the tactile-auditory information would have been integrated instantly with less complex shapes and audio, for example, just one angle or one curve? Less complex shapes would certainly reduce the perceptual load, and thus the attentional load on haptic touch alone, hearing alone, and the two senses together (cf. Jacoby, Hall, \& Mattingley, 2012; Lavie, 1995; Lavie, Lin, Zokaei, \& Toma, 2009; Macdonald \& Lavie, 2011; Sandhu \& Dyson, 2016; Tellinghuisen, Cohen, \& Cooper, 2016; Treisman, 1988, 1995; Treisman \& Paterson, 1984). In fact, perceptual load, and thus attentional load may explain why the participants who were blind in this study ignored the auditory information in Experiment 2. Indeed, according to Graven (2016a), people who are blind are well aware of any attentional load on haptic touch. Again, further research is needed.

In a related vein, could it be that haptic touch and hearing adopted different problem-solving strategies, and that these did not match? Fryer et al. (2014) found that $61 \%$ of their participants, who were visually impaired and who offered a reason for their choice of tactile bouba/kiki $[N=23$. (Total $N=$ 42)], reported sound as their most important problem-solving strategy: 'The name sounded like the feel' (Fryer et al., 2014, p. 169). However, these participants did not necessarily follow the expected association pattern for bouba and kiki, for example, said that 'Kiki sounds like an African name which I associated with crinkly hair' and 'the rounded outline should be "Bouba" because the shape "resembled a boomerang"" [Fryer et al., 2014, p. 170. (Both participants picked out the tactile bouba)]. Considering that the vast majority of these participants had at least some visual imagery (cf. Fryer et al., 2014), could it be that the participants who were blindfolded in this study adopted the same problem-solving strategy for drawing upon their visual imagery [e.g. 'crinkly hair' and 'boomerang' (cf. Experiment 1, pre-test/Trial 1; Experiment 2, Trial 1; Experiment 3, Trial 1)]? Indeed, Graven (2016a) found that people who were able to draw upon visual imagery used a very similar problem-solving strategy for discriminating tactile 2D shapes without hearing, that is, the touch vision strategy - 'I treat them as global shapes' (...) '(...) print " $z$ " is a symbol for all global shapes that resemble the z' (Graven, 2016a, p. 388). When an instant association between the global shape and the print letter did not occur, then 'I recognise them because I count lines' (Graven, 
2016a, p. 389). With these problem solving-strategies in mind, further research is needed to investigate whether people who use haptic touch and who have some visual imagery, in fact, rely on ideaesthesia in order to recognise tactile shapes and/or tactile-auditory regularities (cf. Milan, Iborra, de Cordoba, Juarez-Ramos, Artacho, \& Rubio, 2013).

Furthermore, certain cortical areas are activated regardless of what sensory modality is used to perceive the shape information (known as 'supramodal plasticity'), and with the lack of vision these areas are reorganised; both structurally and functionally [known as 'cross-modal plasticity' (e.g. Amedi, Hofstetter, Maidenbaum, \& Heimler, 2017; Cecchetti, Kupers, Ptito, \& Pietrini, 2016; Rauschecker, 1995; Reich, Szwed, Cohen, \& Amedi, 2011; Sadato, Pascual-Leone, Gradfman, Ibañez, Deiber, Dold, \& Hallett, 1996; Striem-Amit, Cohen, Dehaene, \& Amedi, 2012; Ward \& Meijer, 2010)]. Supramodal plasticity would certainly explain how the participants who were blindfolded in this study were able to draw upon their visual imagery, that is, instantly for every new problem to be solved [(cf. Experiment 1, pre-test/Trial 1; Experiment 2, Trial 1; Experiment 3, Trial 1)] So, why were they not able to draw upon their visual imagery long-term? Could it be that cross-modal plasticity initially creates a state of chaos (cf. Amedi, Merabet, Bermpohl, \& PascualeLeone, 2005; Brunner, 1957; Graven, 2009; Gregory \& Wallace, 1963; Hamilton \& Pascual-Leone, 1998; Hull, 1991; Pascual-Leone \& Torres, 1993; Sacks, 1995; Spence et al. 2001)? Indeed, Experiment 2 found that when the tactile and the auditory information was, or could not be integrated the majority of the participants who were blindfolded sometimes ignored the tactile information, and sometimes the auditory information, as if they were trying out whether to rely on information from haptic touch or on information from hearing - as if they were trying out different problem-solving strategies. In contrast, the participants who were experienced in using haptic touch relied on haptic touch (i.e. the blind-group); and those who were experienced in using vision relied on either vision or hearing [i.e. the vision-group. Cf. Experiment 2)]. Further research is needed to investigate whether people who are fully sighted and who rely on hearing, that is, when the visual and the auditory information is, or cannot be integrated would rely on hearing also when tactile and auditory information is, or cannot be integrated.

Moreover, Graven (2015) found that people who have lost vision recently seem to change problemsolving strategy when they become more experienced in using haptic touch: adopting either the figure identity strategy or the global characteristics strategy (instead of the touch vision strategy). The figure identity strategy performs a specific analysis of the line quantity in each shape, and then recognises the shape according to a set of rules, for example, 'An angle has two lines' and 'When the shape has more than two lines, then it is a curve; not an angle' (Graven, 2016a, p. 387). The global characteristics strategy, in contrast, perceives the global shape. It analyses the global shape only: 'When they have too many lines', for example, it has 'one short and one long' (Graven, 2016a, p. 388). Further research is needed to investigate whether hearing adopts similar problem-solving strategies, and whether haptic touch and hearing adopt similar or different problem-solving strategies for perceiving and processing tactile and auditory information; when this information is presented together. Indeed, could it be that the participants who were blind in this study ignored the auditory information in Experiment 2 because haptic touch and hearing had adopted different problemsolving strategies? Graven (2016b) found that when a problem-solving strategy failed to recognise the tactile shape people still continued using it - they did not adopt a different problem-solving strategy. Further research is needed to investigate whether auditory information affects what problem-solving strategy is adopted for correct and incorrect recognition of tactile shapes.

When taken together, the three experiments in this study suggest that the sensory regularities and mental images concerning bouba and kiki do not have to be based on, or even include visual information. However, further research is needed to fully understand the relationship between haptic touch and hearing in shape recognition. 


\subsubsection{References}

Amedi, A., Hofstetter, S., Maidenbaum, S., \& Heimler, B. (2017). Task Selectivity as a Comprehensive Principle for Brain Organization. Trends in Cognitive Science, 21(5), 307310.

Amedi, A., Merabet, L. B., Bermpohl, F., \& Pascual-Leone, A. (2005). The occipital cortex in the blind: Lessons about plasticity and vision. Current Directions in Psychological Science, 14(6), 306-311.

Bar, M., \& Neta, M. (2006). Humans Prefer Curved Visual Objects. Psychological Science, 17(8), 645-648.

Bertamini, M., Palumbo, L., Gheorghes, T. N., \& Galatsidas, M. (2016). Do observers like curvature or do they dislike angularity? British Journal of Psychology, 107(1), 154-178.

Bremner, A. J., Caparos, S., Davidoff, J., de Fockert, J., Linnell, K. J., \& Spence, C. (2013). "Bouba" and "Kiki" in Namibia? A remote culture make similar sound-shape matches, but different taste-shape matches to Westerners. Cognition, 126(2), 165-172.

Brunner, J. S. (1957). On perceptual readiness. Psychological Review, 64(2), 123-152.

Cecchetti, L., Kupers, R., Ptito, M., \& Pietrini, P. (2016). Are Supramodality and Cross-Modal Plasticity the Yin and Yang of Brain Development? From Blindness to Rehabilitation. Frontiers in Systematic Neuroscience, 10(November, Article 89), 1-8.

Davis, R. (1961). The fitness of names to drawings. A cross-cultural study in Tanganyika. British Journal of Psychology, 52(3), 259-268.

Fontana, F. (2013). Association of Haptic Trajectories to Takete and Maluma. Lecture Notes in Computer Science, 7989, 60-68.

Fryer, L., Freeman, J., \& Pring, L. (2014). Touching words is not enough: How visual experience influences haptic-auditory associations in the "Bouba-Kiki" effect. Cognition, 132(2), 164173.

Graven, T. (2003). Aspects of object recognition: When touch replaces vision as the dominant sense modality. Visual Impairment Research, 5(2), 101-112.

Graven, T. (2004). Recognising tactile representations of familiar objects: The influence of precuing when touch replaces vision as the dominant sense modality. Visual Impairment Research, 6(2-3), 99-110.

Graven, T. (2005). Mental manipulation, thus recognition of familiar shapes: The influence of precuing when touch replaces vision as the dominant sense modality. Visual Impairment Research, 7(2-3), 63-69.

Graven, T. (2009). Seeing Through Touch: When Touch Replaces Vision as the Dominant Sense Modality. Saarbrücken: VDM Verlag Dr. Müller AG \& Co.

Graven, T. (2015). How blind individuals discriminate braille characters: An identification and comparison of three discrimination strategies. British Journal of Visual Impairment, 33(2), $80-95$.

Graven, T. (2016a). How attention is allocated when using haptic touch: Shape feature distinction and discrimination strategy. In F. Bello, H. Kajimoto, \& Y. Visell (Eds.) EuroHaptics 2016, Part 1. Lecture Notes in Computer Science, 9774, (pp. 380-393). Heidelberg: Springer International Publishing AG Switzerland.

Graven, T. (2016b). When the discrimination strategy fails: Revisiting the figure identity strategy, the global characteristics strategy, and the touch vision strategy. British Journal of Visual Impairment, 34(2), 121-129.

Gregory, R. L., \& Wallace, J. G. (1963). Recovering from Early Blindness: A Case Study. Experimental Psychology Society Monograph, 2, 1-46.

Hamilton, R. H., \& Pascual-Leone, A. (1998). Cortical plasticity associated with Braille learning. Trends in Cognitive Sciences, 2(5), 168-174.

Heller, M. A. (1989). Picture and pattern perception in the sighted and the blind: The advantage of the late blind. Perception, 18(3), 379-389.

Heller, M. A., Calcaterra, J. A., Burson, L. L., \& Tyler, L. A. (1996). Tactual picture identification by blind and sighted people: Effects of providing categorical information. Perception \& 
Psychophysics, 58(2), 310-323.

Heller, M. A., \& Gentaz, E. (2014). Psychology of Touch and Blindness. New York and London: Psychology Press.

Heller, M. A., Wilson, K., Steffen, H., Yoneyama, K., \& Brackett, D. D. (2003). Superior haptic perceptual selectivity in late-blind and very-low-vision subjects. Perception, 32(4), 499511.

Hull, J. M. (1991). Touching the Rock. An experience of blindness. London: Arrow Books Limited.

Hötting, K., \& Röder, B. (2004). Hearing Cheats Touch, but Less in Congenitally Blind Than in Sighted Individuals. Psychological Science, 15(1), 60-64.

Hötting, K., \& Röder, B. (2009). Auditory and auditory-tactile processing in congenitally blind humans. Hearing Research, 258(1-2), 165-174.

Jacoby, O., Hall, S. E., \& Mattingley, J. B. (2012). A crossmodal crossover: Opposite effects of visual and auditory perceptual load on steady-state evoked potentials to irrelevant visual stimuli. NeuroImage, 61(4), 1050-1058.

Katz, D. (1989). The world of touch. [Der Aufbau der Tastwelt]. Hillsdale NJ: Lawrence Erlbaum Associates Inc.

Köhler, W. (1929). Gestalt psychology. New York: Liveright.

Lavie, N. (1995). Perceptual Load is a Necessary Condition for Selective Attention. Journal of Experimental Psychology: Human Perception and Performance, 21(3), 451-468.

Lavie, N., Lin, Z., Zokaei, N., \& Toma, V. (2009). The Role of Perceptual Load in Object Recognition. Journal of Experimental Psychology: Human Perception and Performance, 35(5), 1346-1358.

Lederman, S. J., Browse, R. A., \& Klatzky, R. L. (1988). Haptic processing of spatially distributed information. Perception \& Psychophysics, 44(3), 222-232.

Macdonald, J. S. P., \& Lavie, N. (2011). Visual perceptual load induces inattentional deafness. Attention, Perception, \& Psychophysics, 73(6). 1780-1789.

Maurer, D., Pathman, T., \& Mondloch, C. J. (2006). The shape of boubas: sound shape correspondences in toddlers and adults. Developmental Science, 9(3), 316-322.

Milan, E., Iborra, O., de Cordoba, M. J., Juarez-Ramos, V., Artacho, M. R., \& Rubio, J. L. (2013). The Kiki-Bouba Effect A Case of Personification and Ideaesthesia. Journal of Consciousness Studies, 20(1), 84-102.

Millar, S. (1984). Strategy Choices by Young Braille Readers. Perception 13(5), 567-579.

Millar, S. (1997). Reading by touch. London: Routledge.

Millar, S. (2008). Space and Sense. Hove and New York: Psychology Press.

Nielsen, A., \& Rendall, D. (2011). The Sound of Round: Evaluating the Sound-Symbolic Role of Consonants in the Classic Takete-Maluma Phenomenon. Canadian Journal of Experimental Psychology, 65(2), 115-124.

Ostad, S. A. (1989). Mathematics through the fingertips. Basic mathematics for the blind pupil: Development and empirical testing of tactile representations. Hosle: The Norwegian Institute for Special Education.

Ozturk, O., Krehm, M., \& Vouloumanos, A. (2013). Sound-symbolism in infancy: Evidence for sound-shape cross-modal correspondences in 4-months-olds. Journal of Experimental Child Psychology, 114(2), 173-186).

Pascual-Leone, A., \& Torres, F. (1993). Plasticity of the sensorimotor cortex representation of the reading finger in Braille readers. Brain, 116(1), 39-52.

Pathak, K., \& Pring, L. (1989). Tactual picture recognition in congenitally blind and sighted children. Applied Cognitive Psychology, 3(4), 337-350.

Quinn, P. C., Brown, C. R., \& Streppa, M. L. (1997). Perceptual Organization of Complex Visual Configurations by Young Infants. Infant Behavior and Development, 20(1), 35-46

Ramachandran, V. S., \& Hubbard, E. M. (2001). Synaesthesia - A Window Into Perception, Thought and Language. Journal of Consciousness Studies, 8(12), 3-34.

Rauschecker, J. P. (1995). Compensatory plasticity and sensory substitution in the cerebral cortex. 
Trends in Neurosciences, 18(1) 36-43.

Reich, L., Szwed, M., Cohen, L., \& Amedi, A. (2011). A Ventral Visual Stream Reading Center Independent of Visual Experience. Current Biology, 21(5), 363-368.

Sacks, O. (1995). An anthropologist on Mars: seven paradoxical tales. London: Picador

Sadato, N., Pascual-Leone, A., Gradfman, J., Ibañez, V., Deiber, M-P., Dold, G., \& Hallett, M. (1996). Activation of the primary visual cortex by Braille reading blind subjects. Nature, 380(6574), 526-528.

Sandhu, R., \& Dyson, B. J. (2016). Cross-modal perceptual load: the impact of modality and individual differences. Experimental Brain Research, 234(5), 1279-1291.

Silvia, P. J., \& Barona, C. M. (2009). Do people prefer curved objects? Angularity, expertise, and aesthetic preference. Empirical Studies of the Arts, 27(1), 25-42.

Spence, C., Nicholls, M. E. R., \& Driver, J. (2001). The costs of expecting events in the wrong sensory modality. Perception \& Psychophysics, 63(2), 330-336.

Stein, B. E., \& Meredith, M. A. (1993). The Merging of the Senses. Cambridge: The MIT Press.

Striem-Amit, E., Cohen, L., Dehaene, S., \& Amedi, A. (2012). Reading with Sounds: Sensory Substitution Selectivity Activates the Visual Word Form Area in the Blind. Neuron, 76(3), 640-652.

Tellinghuisen, D. J., Cohen, A. J., \& Cooper, N. J. (2016). Now hear this: Inattentional deafness depends on task relatedness. Attention, Perception, \& Psychophysics, 78(8), 2527-2546.

Treisman, A. (1988). Features and objects: The fourteenth Bartlett memorial lecture. Quarterly Journal of Experimental Psychology Section A, 40(2), 201-237.

Treisman, A. (1995). The perception of features and objects. In A. Baddeley \& L. Weiskrantz (Eds.), Attention: Selection, awareness, and control: A tribute to Donald Broadbent (pp. 535). Oxford: Oxford University Press.

Treisman, A. M., \& Paterson, R. (1984). Emergent features, attention, and object perception. Journal of Experimental Psychology: Human Perception and Performance, 10(1), 12-31.

Ward, J., \& Meijer, P. (2010). Visual experiences in the blind induced by an auditory sensory substitution device. Consciousness and Cognition, 19(1), 492-500.

WHO. (2017). ICD-10 version: 2010 (International classification of diseases and related health problems 10th revision). Chapter VII Diseases of the eye adnexa (H00-H59). Retrieved from: http://apps.who.int/classifications/icd10/browse/2016/en\#/H53-H54 